\title{
Another look at semantic priming without awareness
}

\author{
DEAN G. PURCELL \\ Oakland University, Rochester, Michigan \\ ALAN L. STEWART \\ Stevens Institute of Technology, Hoboken, New Jersey \\ and \\ KEITH E. STANOVICH \\ Oakland University, Rochester, Michigan
}

\begin{abstract}
In a recent study, McCauley, Parmelee, Sperber, and Carr (1980) reported results indicating that semantic priming had been produced by visual stimuli that were backward masked at durations too brief for greater than chance report. The conclusions drawn from such an experiment are critically dependent upon whether or not the primes were actually masked below the threshold for identification during priming trials. The three experiments reported here provide evidence that this requirement was not met. Rather, McCauley et al.'s (1980) methodology allowed for an uncontrolled increase in light adaptation during the actual testing of prime efficacy in the priming session. This increase in light adaptation reduced the effectiveness of the backward mask and resulted in an increase in prime visibility during priming trials. Thus, semantic priming probably occurred under conditions in which commensurate visual information was actually available.
\end{abstract}

A stimulus that is too weak to be identified with greater than chance accuracy cannot affect a person's cognitive state, or, at least this became the prevailing view among psychologists following Eriksen's (1960) review of discrimination and learning without awareness. Recent studies challenge this belief (Fowler, Wolford, Slade, \& Tassinary, 1981; Marcel \& Patterson, 1978; McCauley, Parmelee, Sperber, \& Carr, 1980). These studies purport that semantic priming can occur for a tachistoscopically presented stimulus even when the priming stimulus is too weak to be identified by the observer.

As Nolan and Caramazza (1982) have recently suggested (see, also, Merikle, 1982), the terminology used in this research area may have served to obscure the issues. It is generally accepted that performance can be affected by stimuli that are not completely and unambiguously identified. If this is what is meant by priming without awareness, then there is no controversy. The controversial claim is that a certain amount of semantic priming can occur in the

This research was supported by an Oakland University faculty research grant. We thank William $\mathbf{H}$. MacDonald and Sydney Talley for assistance in collecting the data. We also thank Thomas H. Carr for carefully reading an earlier draft of this paper and for his useful comments. D. G. Purcell's mailing address is: Department of Psychology, Oakland University, Rochester, Michigan 48063. absence of evidence that a commensurate amount of sensory processing of the prime has taken place. In particular, the finding that semantic priming can occur under conditions in which the prime is identified at chance accuracy in a forced choice procedure is at the center of much controversy and is the focus of our empirical efforts. We retained the term "awareness" in our title only for consistency with the previous literature and to avoid the cumbersome "semantic priming under conditions in which prime report is at chance accuracy."

The experiments of McCauley et al. (1980) offer the best available evidence for semantic priming without awareness, but they are marred by a subtle, and potentially serious, flaw. It is likely that McCauley et al. (1980) inadvertently light-adapted their subjects during the priming sessions, thereby causing a systematic decrease in their subjects' recognition thresholds. In this case, even accurately assessed thresholds obtained in preliminary trials would have been too liberal to justify their claim to have achieved semantic priming without awareness. If true, this finding proves suspect the best available evidence for semantic priming without awareness.

We have two goals in this paper. The first is to demonstrate that the data of McCauley et al. (1980) can be accounted for by a form of light adaptation and, therefore, do not offer evidence for semantic priming without awareness. Our second goal is to il- 
lustrate the effects of light adaptation in a masking paradigm often used in cognitive psychology. It is not widely known that light adaptation can improve target report under visual masking (Boynton \& Kandel, 1957; Purcell \& Stewart, 1974; Scharf \& Fuld, 1972). Our data will demonstrate the importance of light adaptation when visual masking is used to limit the observer's access to a visual stimulus and will emphasize the necessity to eliminate adaptation effects from cognitive experiments.

To understand our argument with regard to light adaptation, it is necessary to compare McCauley et al.'s (1980) threshold assessment trials with their priming trials. In order to assess the subject's recognition thresholds, McCauley et al. (1980) exposed them to a sequence of a picture stimulus followed by a masking stimulus (In subsequent trials, the picture stimulus was used as a priming stimulus.) The observers were in a darkened room, and before and after each trial they continued to look into the dark viewing box of the tachistoscope. Although the subjects were not dark adapted at the beginning of the assessment trials, they eventually became dark adapted because it took over an hour to assess thresholds for their 10 priming pictures.

During the priming trials, the subjects were presented with the sequence of a priming stimulus followed by a masking stimulus. Unlike the threshold trials, in the priming trials the offset of the mask was followed by a third flash of light, which contained the target stimulus. The target terminated when the subject responded. The target durations ranged from 750 to $800 \mathrm{msec}$. It is our contention that the light from the target flash either prevented dark adaptation from taking place during the priming trials or, at the very least, prevented dark adaptation from proceeding to the level it had reached during the threshold assessment trials.

Assume that the subjects were at a higher level of light adaptation during the priming trials than they were during the threshold assessment trials. Then the masking stimulus would no longer be as effective in suppressing perception of the priming stimulus as it was during threshold assessment. In other words, any threshold fixed under conditions of dark adaptation might allow the subjects to see the priming stimulus under more light-adapted conditions.

Direct evidence for our hypothesis is presented in Experiments 1 and 2. Here, reports of visual stimuli, under conditions of visual backward masking, are compared with reports of the same stimuli when a third stimulus was added to the stimulus train. The third stimulus corresponds to the target stimulus used in McCauley et al.'s (1980) priming trials. Additional, indirect evidence for our hypothesis comes from a third experiment in which priming effects were tested under conditions that minimized adapta- tion differences between the threshold sessions and the priming sessions.

Replicating the essential features of McCauley et al.'s (1980) experiments hinges on another critical methodological point involving threshold assessment. In what they termed their zero-threshold condition, they maintained that no subject was aware of the content of the priming stimuli, which consisted of 10 pictures taken from the Peabody Picture Vocabulary Test. A descending method of limits was used in assessing the zero threshold: The assessment ended with the stimulus duration at which the subject incorrectly identified the stimulus for six consecutive trials. The zero threshold for a picture was achieved by subtracting $5 \mathrm{msec}$ from this duration, "to ensure that the estimate was conservative" (McCauley et al., 1980, p. 268). ${ }^{1}$

McCauley et al.'s (1980) description of the assessment procedure suggests that the subjects were required to make a forced-choice response. McCauley et al. (1980) state that "the subjects were told simply to try to identify the stimulus by naming it aloud and to guess when they were not sure'" (p. 268). Using a forced-choice technique, a subject would have a .47 probability by chance alone of correctly naming a stimulus on one of the six consecutive trials. When we tried to replicate McCauley et al.'s (1980) stated procedure (hereafter called the "reported procedure"'), we sometimes failed to achieve their threshold criterion, even when the stimulus reached 0 duration (see Merikle, 1982). In subsequent discussions, we learned that a forced-choice procedure was not used (Sperber, Note 1). Instead, subjects were allowed not to guess if they said they had not seen the picture. (Hereafter we will refer to this as McCauley et al.'s (1980) "actual procedure"). To insure that we would obtain stable thresholds, however, we used a forced-choice procedure throughout our first experiment.

\section{EXPERIMENT 1}

The object of the experiment was to study the effect of the target flash on an observer's ability to identify a priming stimulus. We hypothesized that the target flash, on a given trial, would light-adapt the visual system, causing a reduction in the effectiveness of the masking stimulus on the succeeding trial. Diminution of masking would be indicated by an increase in the number of correctly reported priming stimuli. Since our interest was only in the light reflected by the target stimulus, no pictorial information was presented in the flash of light corresponding to McCauley et al.'s (1980) target presentation. [To avoid unnecessary repetition, we will refer to McCauley et al. (1980) as M.P.S.C. When a stimulus is substituted for one used by M.P.S.C., we 
will refer to the substitute stimulus as the "mock" stimulus.]

\section{Method}

Our procedures differed from M.P.S.C.'s in that: We used a forced-choice method, we used no pictorial material in the mock target flash, and we used letters as mock priming stimuli. Letters were stimuli with which the observers were familiar and which could be identified by an unambiguous response. Letters also lent themselves to a standard forced-choice procedure in determining the observer's recognition threshold.

Montellesee, Sharpe, and Brown (1979) showed that turning off an adapting light $10 \mathrm{sec}$ prior to testing affected the critical duration for luminance summation. M.P.S.C. report that $10 \mathrm{sec}$ lapsed between their stimulus trials. This suggests that light adaptation from M.P.S.C.'s target might have reduced backward masking of the prime. Since a shorter intertrial interval would bias the experiment in favor of our hypothesis, we carefully separated each trial by $10 \mathrm{sec}$.

Subjects. Four highly trained observers were used. Each had served as an observer in a variety of masking experiments for 3 years or more. Two observers (D.G.P. and A.L.S.) were aware of the hypothesis. Two of the observers were not.

Stimuli. Ten black-on-white uppercase letters (A, H, I, M, T, U, V, W, X, Y) were used to simulate M.P.S.C.'s priming stimuli. The visual angle of the letter set was selected for each subject in a pretest. We chose an angular subtense that produced approximately $40 \%$ correct letter report when followed by a masking stimulus similar to that of McCauley et al. (1980). The letters subtended from .26 to $.53 \mathrm{deg}$. The letters were centered on a point $.5 \mathrm{deg}$ to the right of the point fixated by the subject. The fixation field was dark except for a pin-point-size fixation dot.

The mask was a black-on-white pattern composed of the overlapping, uppercase letters $\mathrm{O}$ and $\mathrm{N}$. The mask was centered on the point of fixation and subtended 3 deg horizontally and $2.55 \mathrm{deg}$ vertically. The stimulus field representing the target stimulus was also centered on the fixation point and consisted of a blank white card.

All stimulus fields were 3 deg square. Their white portions were constant at about $60 \mathrm{~cd} / \mathrm{m}^{2}$. The space-average luminance of the black and white mask was $30 \mathrm{~cd} / \mathrm{m}^{2}$. The duration of the mask was constant at $50 \mathrm{msec}$. The mock target flash was constant at $1 \mathrm{sec}$, and its onset occurred $450 \mathrm{msec}$ after the offset of the mask, the same interstimulus interval used by M.P.S.C.

Toward the end of the practice sessions, the duration of the mock prime was adjusted to bring each observer's performance to approximately $40 \%$ correct letter report. This was done to compensate for practice effects. The final durations were constant for each observer and ranged from 40 to $65 \mathrm{msec}$. The mask onset coincided with the offset of the letters, so that, just as with M.P.S.C., the duration of the mock prime was confounded with the stimulus onset asynchrony of the masks.

Apparatus and Procedure. A four-channel Iconix tachistoscope was employed to present all stimuli. Subjects were tested in $11 / 2-h$ sessions. Prior to testing, they were dark adapted for $10 \mathrm{~min}$. The simulated threshold condition consisted of a letter presentation followed by the mask. The simulated priming condition consisted of a letter presentation, the mask, a dark interval, and the homogeneously illuminated field corresponding to M.P.S.C.'s target field.

The subjects initiated each trial. The order of conditions was counterbalanced across subjects, with half of them receiving the simulated threshold condition first. When the second condition was run, the subjects were given five practice trials, both to familiarize them with the new condition and to stabilize light adaptation at the new level. When changing to the simulated threshold conditions, after running in the simulated priming trials, the subjects were again dark adapted for $7 \mathrm{~min}$. Forty trials were run in each condition, in blocks of 10 trials each.

\section{Results and Discussion}

Observers remarked that it became easier to see the letters during the course of the first five simulated priming trials. No improvement was noticed during the simulated threshold trials. During the experiment, letters were reported correctly on $37.5 \%$ of the simulated threshold trials, and on $78.75 \%$ of the simulated priming trials. The difference of $41 \%$ is statistically significant $[\mathrm{t}(3)=9.13, \mathrm{p}<.01]$.

Superior target report during the simulated priming trials supports our hypothesis that the priming stimuli used by M.P.S.C. were easier to see during the priming sessions than they were during threshold assessment. Although M.P.S.C's subjects did not report priming stimuli under the zero-threshold condition, our results suggest that they might well have been aware of them during M.P.S.C.'s priming trials. We attribute the improvement in our subjects' performance to light adaptation produced by the simulated target flash.

Our finding is consistent with research indicating that backward masking is reduced by light adaptation (Boynton \& Kandel, 1957; Purcell \& Stewart 1974; Scharf \& Fuld, 1972). ${ }^{2}$ We infer that the light of the target flash adapted M.P.S.C.'s subjects, just as it did in our experiment, and produced a similar improvement in their subjects' ability to see the priming stimuli. Their subjects' failure to report the priming stimuli may represent some form of output interference induced by reporting of the target stimuli, although the nature of the interference is not certain. At any rate, M.P.S.C. propose output interference to account for the lower than expected prime report that they observed with all prime durations greater than the zero-threshold duration. With M.P.S.C.'s procedure, output interference would be artificially enhanced, since their subjects were instructed to report the priming stimuli only if they chose to.

\section{EXPERIMENT 2}

Experiment 1 simulated the features of M.P.S.C. that would be expected to affect the subject's light adaptation. A second experiment was designed to see if the differences found between threshold trials and simulated priming trials would appear using a procedure still more closely approximating that of the original study. Accordingly, naive subjects were used, the priming stimuli were line drawings of common objects rather than letters of the alphabet, stimulus thresholds were estimated by using a descending method of limits and M.P.S.C.'s actual procedure, and the threshold and priming trials were held on different days. The goal of Experiment 2 was the same as that of Experiment 1-to determine if subjects could report the priming stimuli more accurately during the priming trials than during threshold assess- 
ment trials. The only significant difference between the experiment conducted by M.P.S.C. and Experiment 2 is that the stimulus corresponding to the target flash was a homogeneously illuminated field.

Given accurate threshold estimates for the priming stimuli, M.P.S.C. would expect that subjects could not identify zero-threshold priming stimuli during priming sessions. Our hypothesis, based on the results of Experiment 1, was that the priming stimuli could be reported during simulated priming trials, even when set at zero-threshold.

\section{Method}

Subjects. Nine naive subjects were recruited from an introductory psychology class.

Stimuli. Stimulus materials consisted of five black-on-white line drawings taken from the Peabody Picture Vocabulary Test. These stimuli ranged in horizontal extent from 1.68 to $2.93 \mathrm{deg}$, and were centered on the subjects' point of fixation. The mask was the same as in Experiment 1. Timing and luminance levels were the same as in Experiment 1, except that the mock target flash was shortened to $800 \mathrm{msec}$, a value close to the average value obtained by M.P.S.C.

Apparntus and Procedure. The general procedure was the same as that described by M.P.S.C., except for the following departure: During the threshold assessment trials, our subjects were told that we were trying to establish a duration at which they could no longer see the picture being flashed before them. They were instructed to name the picture out loud and to guess if they saw only a portion of the picture. They were told that if they saw nothing, they were not to name the picture. These instructions conform to the actual procedure used by M.P.S.C. (Sperber, Note 1).

Once the subjects were dark adapted, the stimulus duration was decreased in $10-\mathrm{msec}$ steps, starting at a duration of $150 \mathrm{msec}$. Threshold assessment ended when the subject incorrectly identified the stimulus on three separate presentations at a given duration. Our criterion for threshold differed from the six separate errors required by M.P.S.C., because we used 5 priming stimuli rather than 10. (Both M.P.S.C.'s and our procedure gave similar expectations of the subjects' making correct responses by chance alone. The point is moot, however, in that neither M.P.S.C. nor we used a forced-choice procedure in determining the prime's threshold.) Just as did M.P.S.C., we obtained the zero-threshold duration by subtracting $5 \mathrm{msec}$ from the criterion threshold. Threshold assessment took about $1 / 2 \mathrm{~h}$, and the average criterion threshold duration was $90.11 \mathrm{msec}$.

The priming session was held the next day. Two prime durations were used: The zero threshold and a duration equal to the zero threshold plus $20 \mathrm{msec}$. This second condition simulated M.P.S.C.'s $2 / 3$ full-threshold condition. This condition was included, in part, because M.P.S.C's $1 / 3$ and $2 / 3$ full-threshold presentations might have served to refresh the subjects' memories of pictures included in the set of priming stimuli and thus to increase the chances of the subjects' recognizing zero-threshold primes.

The priming session differed in two important ways from the threshold sessions. First, just as in Experiment 1, a homogeneous flash of light, simulating the target flash of M.P.S.C., followed the onset of the mask by $450 \mathrm{msec}$. Second, we imposed a different criterion for the subjects' responses. We required that subjects make a forced-choice response on each trial, even if it meant that they had to guess. This differed from the lax criterion imposed by M.P.S.C., but would provide a more sensitive and reliable indicator of the prime information available to a subject on a priming trial.

The subjects were given 15 trials per priming stimulus for each of two threshold durations. Each subject received 150 trials, run in blocks of 10 trials, with each priming stimulus and each threshold duration sampled once per block in a random order. At the begin- ning of each session, the subjects were given one practice trial with each prime set at a duration of $150 \mathrm{msec}$. Just as in M.P.S.C., the subjects were not given a period of explicit dark adaptation and testing began immediately after the practice trials. The intertrial interval was between 10 and $15 \mathrm{sec}^{3}$

Using our procedure, it is clear that the subject's level of light adaptation would be greater during the simulated priming trials than it was during the threshold trials. This is not an oversight; rather, it is the critical feature which ties our procedure to that used by M.P.S.C. M.P.S.C. failed to control for light adaptation, and it is our hypothesis that the increased light adaptation effected by the priming trials allowed their subjects to see the zerothreshold priming stimuli-stimuli which they could not identify under conditions of threshold assessment.

\section{Results and Discussion}

The findings of Experiment 1 were confirmed. Subjects correctly reported the primes on $69.58 \%$ of the zero-threshold priming trials. By chance alone, they would have been expected to name the stimuli correctly on $20 \%$ of the trials. The improvement of $49 \%$ is statistically significant by a single-means $t$ test (Hays, 1973) $[\mathrm{t}(8)=7.85, \mathrm{p}<.001]$. Average scores for individual subjects ranged from $94.4 \%$ to $28.0 \%$ correct, as can be seen in Table 1 . In the $2 / 3$ fullthreshold priming trials (the zero threshold plus $20 \mathrm{msec}$ ), the subjects averaged $87.13 \%$ correct report. This is $20.46 \%$ greater than the expected $66.67 \%$ correct. This improvement is also statistically significant by means of a t test $[\mathrm{t}(8)=4.63, \mathrm{p}<$ $.02]$. Average scores ranged from $100 \%$ to $48 \%$ correct.

We did not determine the actual $2 / 3$ full-threshold duration of each subject, but estimated it from the available data. Accordingly, we place less importance on the data of the simulated $2 / 3$ full-threshold condition. In Experiment 2, we compared our subjects' performance to that expected by M.P.S.C. Alternatively, a comparison can be made between the performance of our subjects under the zero-threshold condition and the performance of M.P.S.C.'s subjects at a comparable stimulus duration. Our subjects' average zero-threshold duration was $90.11 \mathrm{msec}$, which falls between the zero threshold and $1 / 3$ fullthreshold durations of M.P.S.C. Our subjects' performance on priming trials, under the zero-threshold condition (69.58\%), surpassed that expected under the $1 / 3$ full-threshold condition of M.P.S.C. The difference was statistically significant $[\mathrm{t}(8)=5.59, \mathrm{p}<$ .0011 .

The conditions of Experiment 2 more closely approximated those reported by M.P.S.C. than did Experiment 1 . Experiment 2 is confounded in that both the strictness of the requirement to report the prime and the adaptation level varied between the threshold and prime trials. M.P.S.C.'s study also confounded these variables, but with the requirement to report the prime relaxed on priming trials. The use of a strict criterion in Experiment 2 enabled us to demonstrate that M.P.S.C.'s subjects had more informa- 
Table 1

Percent Correct Report of the Zero-Threshold, and the Zero-Threshold Plus $20 \mathrm{Msec}$, Mock Primes

\begin{tabular}{|c|c|c|c|c|c|c|c|c|c|}
\hline \multirow{2}{*}{$\begin{array}{c}\text { Prime } \\
\text { Duration }\end{array}$} & \multicolumn{9}{|c|}{ Subject } \\
\hline & 1 & 2 & 3 & 4 . & 5 & 6 & 7 & 8 & 9 \\
\hline Zero & 90.00 & 89.00 & 94.00 & 85.40 & 53.20 & 30.80 & 61.33 & 93.33 & 28.80 \\
\hline Zero +20 & 97.50 & 100.00 & 98.67 & 85.33 & 89.33 & 65.33 & 97.33 & 98.67 & 48.00 \\
\hline
\end{tabular}

tion regarding primes during priming trials than M.P.S.C.'s experiment indicated. Although the high levels of prime report in the priming session of Experiment 2 might be due solely to the criterion shift between threshold and priming trials, it is more likely that it is due to the change in adaptation level. This contention is supported by the adaptation effects shown in Experiment 1, in which criterion was held constant between the simulated threshold and priming trials. The results of Experiments 1 and 2 indicate that a plausible explanation for M.P.S.C's purported demonstration of semantic priming without awareness is that their subjects extracted significant visual information in the zero-threshold priming condition. However, this fact was obscured by a combination of output interference and lax requirement for prime report.

\section{EXPERIMENT 3}

In order to control for the sensory effects induced by the target flash, we reran M.P.S.C.'s experiment with all stimuli superimposed on a continuously illuminated background. The transient illumination afforded by the target stimulus is negligible relative to a strong adapting background and, therefore, contributes little to a subject's light adaptation. As a result, the threshold assessment trials and the priming trials could be considered to take place under equivalent levels of light adaptation. If we could still obtain semantic priming with zero-threshold primes when adaptation was constant across all conditions, then the conclusion of M.P.S.C. would be supported. If, however, our zero-threshold stimuli failed to produce semantic priming, it is likely that M.P.S.C.'s original experiment confounded type of trial (threshold vs. priming) with adaptation level.

\section{Method}

Based on the results of Fischler and Goodman (1978), we introduced a procedural change designed to increase the likelihood of detecting semantic priming. One group of subjects was not required to name the priming stimuli during the priming trials. We called this group the No-Prime/Name group. A second group was asked to name both the priming stimuli and the target stimuli, just as M.P.S.C's subjects had been. Aside from the fact that they were light adapted during both the threshold assessment and the semantic priming trials, this Prime/Name group was tested under the same conditions as were M.P.S.C.'s subjects.

Subjects. Nineteen new subjects were recruited from an introductory psychology class. Eight served in the No-Prime/Name group and 11 served in the Prime/Name group.
Stimuli. Stimuli were similar to those of Experiment 2, with the following exceptions: Ten pictures were used as primes, and each picture was paired with a second stimulus. The second stimulus was used as the target in the priming trials. Each prime was paired with a semantically related as well as semantically unrelated target producing 20 combinations of primes and targets.

The target was exposed for $2 \mathrm{sec}$. Both the priming stimuli and the target stimuli were superimposed on a $45-\mathrm{cd} / \mathrm{m}^{2}$ adaptation field. The field was illuminated throughout both the threshold assessment and the priming trials. The adaptation field also contained a small black fixation point which was positioned to fall in the center of the stimulus field. To maintain a constant level of light adaptation, the experiment was run in an illuminated room whose walls reflected approximately $48 \mathrm{~cd} / \mathrm{m}^{2}$.

Apparatus and Procedure. We used the same apparatus as in Experiments 1 and 2 . In addition, the tachistoscope was interfaced with a voice-operated relay and a timer accurate to within $1 \mathrm{msec}$. The voice-operated relay allowed accurate measurement of the interval between the onset of the target stimulus and a subject's spoken response.

In addition to semantic relatedness, we investigated two exposure durations for the priming stimulus. Primes were exposed for a zero-threshold duration, predetermined for each subject in a separate set of assessment trials, and for a $250-$ msec duration. The zero-threshold condition was the test condition for establishing the occurrence of semantic priming without awareness. The 250 -msec duration was essentially a control condition like the suprathreshold condition of M.P.S.C. and was designed to provide subjects with a prime presented at a duration that would allow easy identification. It was included to determine whether our procedures would produce semantic priming in the event that no priming effect was observed with the zero-threshold priming stimuli.

During the threshoid session, the initial stimulus exposure was $100 \mathrm{msec}$. The duration was decreased by $10 \mathrm{msec}$ each time the subject correctly named the stimulus. Trials continued at any given duration until the subject had made six consecutive errors. The zero-threshold durations were determined in a single hourlong session.

The instructions read to the subjects in the Prime/Name group resulted in a somewhat lax criterion for reporting the primes on the threshold assessment trials. The subjects were instructed to name the primes if they could, or to indicate that they could not see them well enough to make a good guess. This procedural change was an error on the part of an experimenter who read the subjects the instructions for the priming trials instead of the instructions for the threshold trials. While this error prevented us from replicating the reported procedure of M.P.S.C. it proved to be a close approximation to their actual procedure. The zero-threshold duration for the Prime/Name group averaged $25.89 \mathrm{msec}$.

The eight subjects in the No-Prime/Name group were given a forced-choice task during threshold assessment. They were instructed to name the picture stimulus even if they felt that they had not seen the stimulus. They were not allowed to simply say that they had not seen anything. We employed this forced-choice procedure in conjunction with the strict criterion of six consecutive errors because we thought, at the time, that it was consistent with M.P.S.C.'s reported procedure. In accordance with Merikle's (1982) analysis of M.P.S.C., this procedure led to zero-threshold values of zero duration for some stimuli. Since we did not want to turn the experiment into an investigation of extrasensory perception, we assigned a 5-msec duration to any priming stimulus that 
reached zero duration. About one-quarter of the priming stimuli were affected by application of this rule. We decided not to include these stimuli in the data analysis when we learned that M.P.S.C. had not used a forced-choice procedure in their assessment trials. It is likely that 5-msec priming stimuli are too brief to have mediated semantic priming, even under the most favorable conditions (see M.P.S.C. Experiment 2). Including such brief stimuli would only bias the data in favor of our hypothesis.

Priming stimuli were presented at their zero-threshold duration or at a duration of $250 \mathrm{msec}$. In addition, a homogeneously illuminated field of $50 \mathrm{msec}$ duration was substituted for a priming stimulus on some trials (the no-prime condition). Each subject was given a total of 60 trials in a single 1 -h session. These trials were divided into 10 blocks of six trials each. Each block contained a related priming stimulus and an unrelated priming stimulus, each at two different durations, as well as two no-prime trials. The order of stimulus presentation was random, with the constraints that no stimulus be presented twice in a row and that each condition occur within each block. Twelve practice trials were given at the beginning of each session.

\section{Results and Discussion}

Target pictures were named accurately, with errors made on fewer than $1 \%$ of the trials, just as in M.P.S.C. The Prime/Name group correctly reported the priming stimuli on only $0.45 \%$ of the zerothreshold priming trials, but correctly reported them on $96.82 \%$ of the trials when the stimulus duration was $250 \mathrm{msec}$. The factors of relatedness (target stimuli were either related or unrelated to the priming stimuli) and the duration of the priming stimuli (zero-threshold duration or $250 \mathrm{msec}$ ) were within subjects. The requirement to report the prime (Prime/Name vs. No-Prime/Name) was a betweensubjects factor. The results for these conditions are presented in Table 2.

The effect of prime report was statistically significant $[F(1,17)=14.81, p<.002]$. The latency for naming the target was, on the average, $191 \mathrm{msec}$ shorter for subjects in the No-Prime/Name group (604 msec) than in the Prime/Name group (795 msec). This result is consistent with Fischler and Goodman (1978) and supports their argument that having to recall the priming stimulus requires additional processing of information by the subject. ${ }^{4}$ The following interactions and effects were not statistically significant: group membership $\times$ the duration of the priming stimulus $[F(1,17)=2.02]$, group membership $\times$ relatedness $[F(1,17)=.22]$, groups $\times$ prime duration $\times$ relatedness $[\mathrm{F}(1,17)=.11]$, prime duration $[\mathrm{F}(1,17)=$ $3.62]$, and semantic relatedness $[\mathrm{F}(1,17)=.45]$.

The duration $\times$ semantic relatedness interaction was statistically significant $[F(1,17)=13.06, p<.005]$. Planned comparisons, based on the mean scores, collapsed across groups, demonstrated that semantic relatedness was an effective variable only with a 250msec-duration priming stimulus $[\mathrm{F}(1,17)=9.98, \mathrm{p}<$ $.01]$.

The failure to find an overall effect of semantic relatedness is inconsistent with the findings of M.P.S.C. While the zero-threshold-duration primes gave no indication that semantic priming had occurred, the
Table 2

Mean Response Times (in Milliseconds) for Pictures Preceded by Related and Unrelated Primes

\begin{tabular}{lccc}
\hline & \multicolumn{2}{c}{ Duration } \\
\cline { 2 - 3 } \multicolumn{1}{c}{ Prime } & 250 & $\begin{array}{c}\text { Zero- } \\
\text { Threshold }\end{array}$ \\
\hline & Unreiated & \\
No Prime Name & 633 & 589 \\
Prime Name & 850 & 744 \\
Mean & 742 & 667 \\
& Related & & \\
No Prime Name & & 581 & 613 \\
Prime Name & 815 & 773 \\
Mean & 698 & 693 \\
Mean Priming Effect & $44^{*}$ & -26 \\
\hline
\end{tabular}

Note-For No Prime trials the RT for No Prime Name groups was 600 msec and for Prime Name groups, 777 msec. Value significant at $p<.01$ by planned comparison indicated by asterisk.

stimuli presented at a duration of $250 \mathrm{msec}$ did yield semantic priming with our subjects. Our failure to obtain semantic priming with zero-thresholdduration primes indicates that a properly assessed and stable zero-threshold duration is too brief to produce semantic priming. In other words, what is too brief to be recognized by an observer is too brief to produce semantic priming.

Experiment 3 indicates that M.P.S.C. may well have determined conservative threshold estimates for their priming stimuli on threshold trials, thus protecting themselves from the methodological objections raised by Merikle (1982). But M.P.S.C.'s failure to equalize their subjects' light adaptation across all experimental conditions is a serious methodological flaw. Even when properly assessed, a recognition threshold set during dark-adapted threshold trials is too liberal to prevent a subject from extracting some visual information from the prime stimulus on lightadapted priming trials. A clear, although perhaps extreme, example of the power of light adaptation with this masking paradigm is shown by comparing the light-adapted Experiment 3 threshold values with the dark-adapted threshold values of Experiment 2. In light-adapted Experiment 3, the No-Prime/Name group gave an average prime threshold of $24.21 \mathrm{msec}$ and the Prime/Name group gave a threshold of $25.89 \mathrm{msec}$. In contrast to this, the dark-adapted conditions of Experiment 2 gave an average threshold value of $90.11 \mathrm{msec}$.

\section{GENERAL DISCUSSION}

McCauley et al. (1980) concluded that "semantic priming effects can be obtained with pictures at prime exposure durations too brief for conscious identification of the prime to occur" (p. 265). This conclusion may have been premature. It is possible that light adaptation may have contributed to the en- 
hanced performance which McCauley et al. (1980) observed in their priming sessions. Experiment 3 deviated from the experiment of McCauley et al. (1980) in just one respect-all stimuli were presented against an illuminated background field. The tachistoscope used by McCauley et al. (1980) lacked the additional stimulus field that our experiment required. But, both Experiment 1 and Experiment 2 indicate that light adaptation across all experimental conditions is critical if sensory effects are to be controlled. Controlling for light adaptation, we produced semantic priming, but only with priming stimuli above the subjects' recognition thresholds. It is clear, therefore, that if McCauley et al.'s (1980) tachistoscope had afforded them a lighted fixation field and if they had equated the sensory state of their subjects across all experimental conditions, they probably would not have produced semantic priming with their zero-threshold-duration stimuli.

A review of the literature on semantic priming without awareness is outside the scope of this paper, but an additional comment is in order. Merikle's (1982) criticism of McCauley et al. (1980) is irrelevant, because it is directed at their reported procedure rather than their actual procedure. However, our findings vitiate McCauley et al.'s (1980) claim to have produced semantic priming in the absence of commensurate visual information. Finally, it should be noted that Fowler et al. (1981) failed to replicate their own evidence for semantic priming without awareness. In their Experiment 6, Fowler et al. (1981) found no indication of semantic priming when the stimulus onset asynchrony between the priming stimulus and the target was 200 msec. In a second condition, one in which the stimulus onset asynchrony was $2 \mathrm{sec}$, reaction time to a word was decreased by $33 \mathrm{msec}$ under conditions of semantic priming. However, an error rate difference of $5 \%$ in the opposite direction indicates a possible speedaccuracy tradeoff. Recently, Nolan and Caramazza (1982) reported that they were unable to replicate Marcel and Patterson (1978). These results, the methodological issues raised by Merikle (1982), and our data, in combination, demonstrate that the evidence for semantic priming from subthreshold stimuli is equivocal.

\section{REFERENCE NOTE}

1. R. D. Sperber, personal communciation, 1981. visual system as a function of adaptation level. Journal of the Optical Society of America, 1957, 47, 275-286.

ERIKSEN, C. W. Discrimination and learning without awareness: A methodological survey and evaluation. Psychological Review, 1960, 67, 279-300.

Fischler, I., \& Goodman, G. O. Latency of associative activation in memory. Journal of Experimental Psychology: Human Perception and Performance, 1978, 4, 455-470.

Fowler, C. A., Wolford, G., Slade, R., \& Tassinary, L. Lexical access with and without awareness. Journal of Experimental Psychology: General, 1981, 110, 341-362.

HAYs, W. L. Statistics for the social sciences. New York: Holt, Rinehart and Winston, 1973.

Marcel, A. J., \& Patterson, K. E. Word recognition and production: Reciprocity in clinical and normal studies. In J. Requin (Ed.), Attention and performance, VII. Hillsdale, N.J: Erlbaum, 1978.

McCauley, C., Parmelee, C. M., Sperber, R. D., \& Cark, T. H. Early extraction of meaning from pictures and its relation to conscious identification. Journal of Experimental Psychology: Human Perception and Performance, 1980, 6, 265-276.

Merikle, P. M. Unconscious perception revisited. Perception \& Psychophysics, 1982, 31, 298-301.

Montellese, S., Sharpe, L. T., \& Brown, J. L. Changes in critical duration during dark-adaptation. Vision Research, 1979, $19,1147-1153$

Nolan, K. A., \& Caramazza, A. Unconscious perception of meaning: A failure to replicate. Bulletin of the Psychonomic Society, 1982, 20, 23-26.

Purcell, D. G., \& Stewart, A. L. Backward masking by pattern mask: Effect of adaptation and target energy. Bulletin of the Psychonomic Society, 1974, 3, 137-138.

SchARF, B., \& FULD, K. Reduction of visual masking by a priming flash. Journal of Experimental Psychology, 1972, 94, 116-118.

\section{NOTES}

1. Just how conservative this procedure was could only have been determined if McCauley et al. (1980) had established the slope of the psychometric function relating stimulus report to stimulus duration.

2. The results of Experiment 1 also suggest that the findings of Montellese, Sharpe, and Brown (1979), regarding the time course of dark adaptation and its effects on the critical duration, will generalize to visual backward masking.

3. Subjects were given a 3-min rest period halfway through the session. After the rest interval, the subjects were given five warmup trials in which they viewed a train of homogeneous flashes whose timing and luminance were identical with those of a priming trial. This was done to stabilize the subjects' adaptation level.

4. Reaction time (RT) was faster when subjects were not asked to name the prime and slower when they were asked to name the prime. But increases in RT were found with both the zero-threshold condition and in the no-prime conditions, when no priming stimuli were seen. Therefore, increases in RT might be related to a subject's expectation that he or she will be asked to name the prime, rather than to output interference or some other memory-related phenomenon.

\section{REFERENCES}

Boynton, R. M., \& Kandel, G. On responses in the human
(Manuscript received October 25, 1982; revision accepted for publication March 8, 1983.) 